\title{
Quasi-total Graphs with Crossing Numbers
}

\author{
V. R. Kulli ${ }^{1}$, B. Basavanagoud ${ }^{2}$ and K. M. Niranjan ${ }^{3}$ \\ ${ }^{1}$ Department of Mathematics, Gulbarga University, Gulbarga-585106, Karnataka, India \\ ${ }^{2}$ Department of Mathematics, Karnataka University, Dharwad, Karnataka, India \\ ${ }^{3}$ Department of Computer Science and Engineering, S.J.M.I.T, NH4 Bye Pass Road, \\ Chitradurga-577 502, Karnataka, India
}

Received 9 October 2009, accepted in final revised form 25 March 2010

\begin{abstract}
We establish here necessary and sufficient conditions for quasi-total graphs to have crossing numbers $k(k=1,2$ or 3$)$.

Keywords: Quasi-total; Crossing numbers.

C 2010 JSR Publications. ISSN: 2070-0237 (Print); 2070-0245 (Online). All rights reserved.

DOI: 10.3329 /jsr.v2i2.3527

J. Sci. Res. 2 (2), 257-263 (2010)
\end{abstract}

\section{Introduction}

All graphs considered here are finite, undirected and without loops or multiple lines. We use the terminology of Harary [1]. A graph is planar if it can be drawn on the plane in such a way that no two of its lines intersect. The crossing number $\operatorname{Cr}(G)$ of a graph $G$ is the minimum number of pair wise intersections of its lines when $G$ is drawn in the plane. Obviously, $\operatorname{Cr}(G)=0$ if and only if $G$ is planar. A graph $G$ has crossing number 1, if $\operatorname{Cr}(G)=1$.

The quasi-total graph $P(G)$ of a graph $G$ is the graph whose point set is $V(G) U X(G)$ and two points are adjacent if and only if they correspond to two non adjacent points of $G$ or to two adjacent lines of $G$ or one is a point and other is a line incident with it in $G$. This concept was introduced in [2].

The following will be useful in the proof of our results.

Remark 1 [3]. For any connected graph $G$, the middle graph $M(G)$ is a spanning subgraph of $P(G)$.

\footnotetext{
${ }^{3}$ Corresponding author: niru_km@yahoo.com
} 
Theorem A [4]. The quasi-total graph $P(G)$ of a graph $G$ is planar if and only if $G$ is of order $\leq 4$.

Theorem $B$ [5]. If a graph $G$ has at least one non-cutpoint of degree 4, then $\mathrm{Cr}(\mathrm{M}(\mathrm{G})) \geq 3$.

Theorem $C$ [5]. Every non-planar graph has a middle graph with crossing number at least 8 .

\section{Main Results}

Fig. 1 shows the connected graph (a) and its quasi-total graph (b) with one crossing. As the graph in Fig. 1(a) is connected, Theorem 1 and Theorem 2 in the paper [1] are incorrect. Theorem 1 of [1] states that the quasi-total graph $P(G)$ of a connected graph $G$ never has crossing number 1 and Theorem 2 of [1] states that the quasi-total graph $P(G)$ of a connected graph $G$ has crossing number 2 if and only if (1) or (2) or (3) holds.

1) $G$ is a path with 5 points

2) $G$ is a path of length two together with two end lines adjoined to some endpoint.

3) $G$ is a path of length two together with a triangle adjoined to some endpoint.

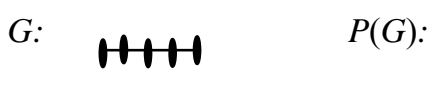

(a)

Fig. 1

(b)

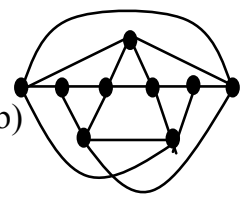

Theorem 1 gives necessary and sufficient condition for the quasi-total graph of a graph with crossing number one.

Theorem 1. The quasi-total graph $P(G)$ of a graph $\mathrm{G}$ has crossing number 1 if and only if $G$ is of order 5 such that every connected component of $G$ is either a path or a triangle.

Proof. Suppose $G$ is a graph satisfying the above condition. Then by Theorem A, $P(G)$ has crossing number at least 1 . We now show that its crossing number is at most 1 . Now the graphs satisfying the condition of the theorem are shown in Fig 2. Then it is easy to see that in an optimal drawing of $P(G)$, it has crossing number one.

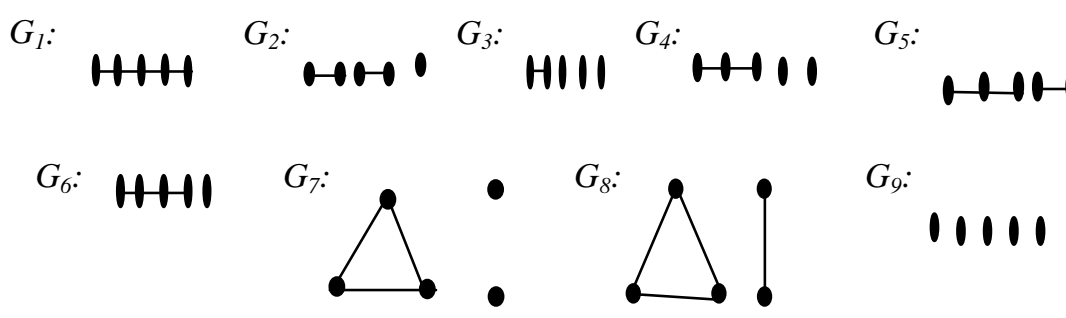

Fig. 2 
Conversely, suppose $P(G)$ has crossing number 1 . Assume $G$ is a graph with at most 4 points. Then obviously $P(G)$ is planar, a contradiction.

Suppose $G$ is a graph with 6 points and assume $\Delta(G) \leq 2$. Then the graphs satisfying the condition are shown in Fig. 3. Then it is easy to see that in an optimal drawing of $P(G)$, it has crossing number more than 1 , which contradicts the hypothesis.

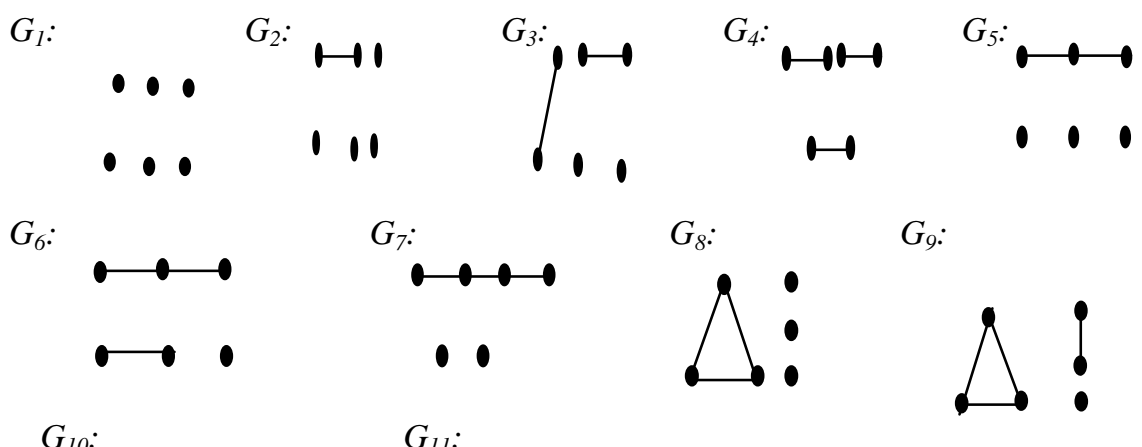

\section{H+HI}

Fig. 3

Suppose $G$ is a graph with 5 points and assume $\Delta(G) \leq 4$. We consider the following cases.

Case 1. Suppose $G$ is non-planar. Then by Theorem $\mathrm{C}$ and Remark $1, P(G)$ has crossing number at least 8 , a contradiction.

Case 2. Suppose $G$ has at least one non-cutpoint of degree 4. Then by Theorem B and Remark 1, $\operatorname{Cr}(P(G)) \geq 3$, a contradiction.

In all the above cases we have a contradiction. This proves that $\Delta(G) \leq 3$.

Suppose $G$ is a graph with 5 points and assume $\Delta(G)=3$. Then the graphs satisfying these conditions are shown in Fig. 4. Then it is easy to see that $\operatorname{Cr}(P(G)) \geq 3$. Thus $\Delta(G) \leq 2$.
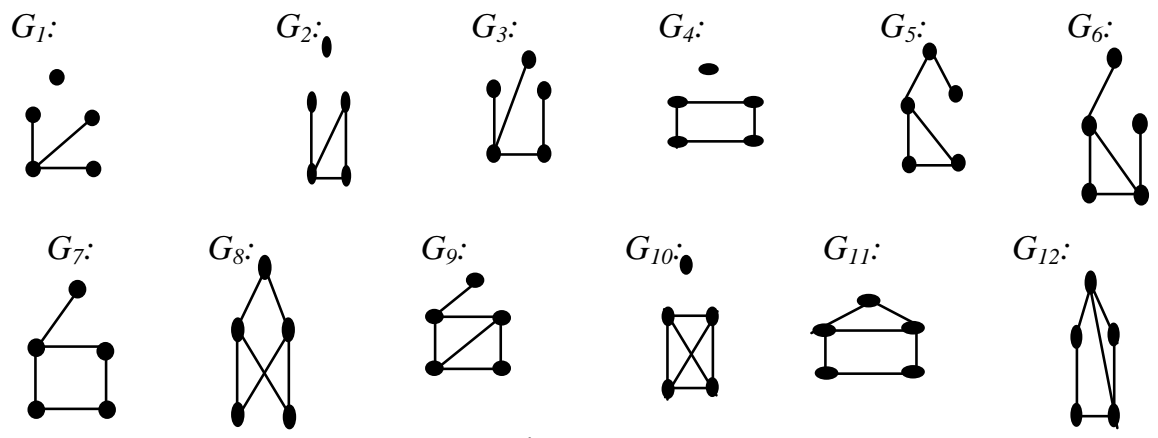

$G_{11}$ :

$G_{12}$ :

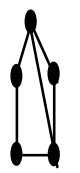

Fig. 4 
Now we consider the following cases:

Case 1. Suppose $G$ is connected. Then $G$ is either a path or a cycle. Suppose $G$ is a cycle. Then $P(G)$ has 3 crossings, a contradiction. Thus $G$ is a path of length four.

Case 2. Suppose $G$ is disconnected. Then every connected component of $G$ is either a path or a triangle. From the above cases, we conclude that $G$ satisfies the condition. This completes the proof.

Corollary 1.1. The quasi-total graph $P(G)$ of a connected graph $G$ has crossing number 1 if and only if $G$ is $P_{5}$.

Theorem 2 gives necessary and sufficient condition for the quasi-total graph of a graph with crossing number two.

Theorem 2. The quasi-total graph $P(G)$ of a graph $G$ has crossing number 2 if and only if $G$ holds either (1) or (2).

1. $G$ is a connected graph of order 5 having a unique cut point of degree 2 and 3 respectively.

2. $G$ is a disconnected graph of order 5 having an isolated point such that the connected component of $G$ has a unique cut point of degree 3 .

Proof. Suppose $G$ holds (1) or (2). Then $G$ is of order 5. Therefore by Theorem A, crossing number of $P(G)$ is at least 1 , since $G$ has a point of degree 3. Therefore $\Delta(G)=3$. Then by Theorem 1, crossing number of $P(G)$ is at least 2 .

Now the graphs satisfying the above condition are shown in Fig 5. Then it is easy to see that in an optimal drawing of $\mathrm{P}(\mathrm{G})$, there are exactly 2 crossings.

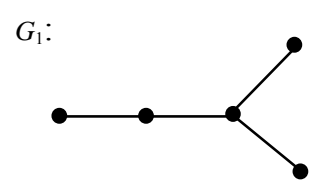

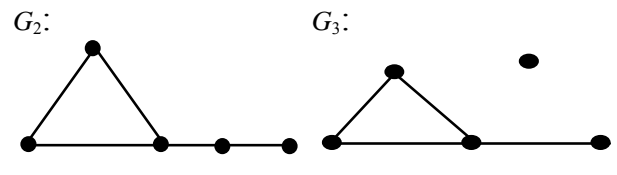

Fig. 5

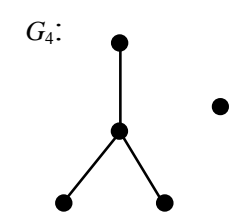

Conversely, suppose $\mathrm{P}(\mathrm{G})$ has crossing number 2 . Assume $G$ is a graph with at most 4 points. Then by Theorem A, $\mathrm{P}(\mathrm{G})$ is planar, a contradiction. Therefore $G$ is of order at least 5.

Case 1. Assume $G$ is a connected graph of order 5. We consider the following subcases.

Subcase 1.1. Assume $G$ is a tree. Suppose $\Delta(G) \leq 2$. Then $G$ is a path of length four and by Theorem 1, $\operatorname{Cr}(P(G))=1$, a contradiction. From the above cases we conclude that $G$ is a path of length three together with an end line adjoined to some non-endpoint.

Subcase 1.2. Assume $G$ is not a tree. We consider the following subcases. 
Subcase 1.2.1. Suppose $\Delta(G)=2$. Then $G$ is $C_{5}$. In an optimal drawing of $P(G)$, it has 3 crossings, a contradiction.

Subcase 1.2.2. Suppose $G$ has at least two points of degree 3. Then it is easy to observe that $G$ is a cycle of length three together with two end lines. Thus $P(G)$ has 3 crossings, a contradiction.

Subcase 1.2.3. Suppose $G$ has exactly one point of degree 3 . Then clearly $G$ is a cycle of length four together with an end line adjoined to some point. Then it easy to see that $P(G)$ has at least 3 crossings, again a contradiction. From the above cases we conclude that $G$ is a triangle together with a path of length two adjoined at some point.

Case 2. Assume $\mathrm{G}$ is a disconnected graph of order 5. We consider the following subcases.

Subcase 2.1. Assume the connected component of $G$ is a tree. Suppose $G$ has exactly one isolated point with $\Delta(G) \leq 2$. Then clearly $G$ is a path of length three with an isolated point and by Theorem $1, \operatorname{Cr}(P(G))=1$, a contradiction. From the above cases we conclude that $G$ has a unique cut point of degree 3 with one isolated point. That is clearly $G$ is $K_{1,3}$ with exactly one isolated point.

Subcase 2.2. Assume the connected component of $G$ is not a tree. Suppose $\Delta(G) \leq 2$. Then clearly, $G$ is a triangle with two isolated points and by Theorem $1, \operatorname{Cr}(P(G))=1$, again a contradiction. From the above cases, we conclude that $G$ is a path of length one together with a triangle adjoined to some end point with exactly one isolated point. From the above cases, we conclude that $G$ satisfies the condition. This completes the proof.

Corollary 2.1. The quasi-total graph $P(G)$ of a connected graph $G$ has crossing number 2 if and only if $G$ is of order 5 having a unique cutpoint of degree 2 and 3 respectively.

We now give a characterization of quasi-total graphs with crossing number 3 .

Theorem 3. The quasi-total graph $P(G)$ of a graph $G$ has crossing number 3 if and only if $G$ is either (1) or (2) or (3).

1. $G$ is $K_{1,4}$ or $C_{5}$ or a triangle together with two end line adjoined at different points.

2. $G$ has an isolated point and the connected component of $G$ is $K_{4}-x$.

3. $G$ is of order 6 such that every connected component of $G$ is either a path or a triangle, except the graph $P_{5} \cup K_{1}$.

Proof. Suppose $G$ is a graph satisfying (1) or (2) or (3). Then by Theorem 1 and Theorem $2, P(G)$ has crossing number at least 3 . We now show that its crossing number is at most 3.

Suppose $G$ satisfies condition (1). Then the graphs satisfying the condition (1) are shown in Fig. 6. Then $P(G)$ has crossing number 3 . 
Suppose $G$ satisfies condition (2). Then the graphs satisfying the condition (2) are shown in Fig. 7. Then $P(G)$ has crossing number 3.

Suppose $G$ satisfies condition (3). Then the graphs satisfying the condition (3) are shown in Fig. 3. Then $P(G)$ has crossing number 3.
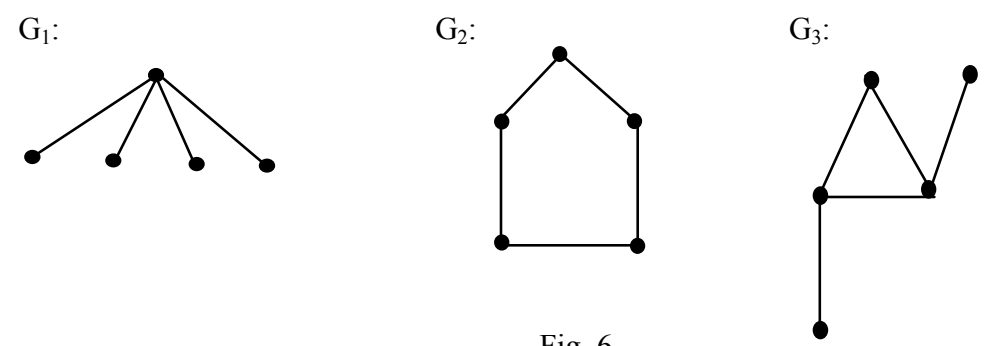

Fig. 6

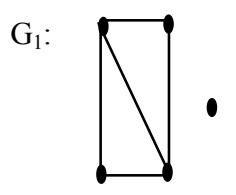

Fig. 7

Conversely, suppose the quasi-total graph $P(G)$ of a graph $G$ has crossing number 3.Then it is non-planar. By Theorem A, $G$ is a graph with at least 5 points.

Case 1. Assume $G$ is a connected graph of order 5. We consider the following subcases, Subcase 1.1. Assume $G$ is a tree. We consider the following subcases

Subcase 1.1.1. Suppose $\Delta(G) \leq 2$. Then $G$ is a path and by Theorem $1, \operatorname{Cr}(P(G))=1$, a contradiction.

Subcase 1.1.2. Suppose $\Delta(G)=3$. Then clearly $G$ is a path of length three together with an end line adjoined to some non-end point. Then by Theorem 2, $\operatorname{Cr}(P(G))=2$, again a contradiction.

Subcase 1.2. Assume $G$ is not a tree. We consider the following subcases.

Subcase 1.2.1. Suppose $G$ has exactly one point of degree 3. Then $G$ is a cycle of length four together with an end line adjoined to some point. Then $\operatorname{Cr}(P(G))=4$, a contradiction.

Subcase 1.2.2. Suppose $G$ has exactly one point of degree 3. Then $G$ is a triangle together with a path of length 2 adjoined at some point. Then it is easy to see that the crossing number of $P(G)$ is at least 4 , again a contradiction.

Subcase 1.2.3. Suppose $\Delta(G) \leq 4$. Then clearly $G$ has at least 2 points of degree 2 and one point of degree 3, 4 and 1 . Then, $P(G)$ has crossing number 4 . From the above cases we conclude that $G$ holds (1). 
Case 2. Assume $G$ is a disconnected graph of order five. We consider the following subcases.

Subcase 2.1. Assume the connected component of $G$ is a tree. Then every connected component of $G$ is a path. Then by Theorem 1 and Theorem $2, \operatorname{Cr}(P(G)) \leq 2$, a contradiction.

Subcase 2.2. Assume the connected component of $G$ is not a tree. We consider the following subcases.

Subcase 2.2.1. Suppose $G$ has at least 3 points of degree 2 and one point of degree 3 and 1 with an isolated point. Then $P(G)$ has crossing number 4 .

Subcase 2.2.2. Suppose $G$ has at least two points of degree 3 and 2 and also one point is of degree 1 . Then $P(G)$ has crossing number 4 . From the above cases, we conclude that $G$ holds (2).

Case 3. Assuming $G$ is a disconnected graph of order 6 , we consider the following subcases.

Subcase 3.1. If we assume the connected component of $G$ is a tree, then every connected component of $G$ is a path.

Subcase 3.2. If we assume the connected component of $G$ is not a tree, then every connected component of $G$ is a triangle. From the above cases, we conclude that $G$ holds (3). This completes the proof.

\section{Conclusion}

We establish here necessary and sufficient conditions for quasi-total graphs to have crossing numbers $k(k=1,2$ or 3$)$. We further find necessary and sufficient conditions for quasi-total graphs to have forbidden sub graphs for crossing numbers $k(k=1,2$ or 3 ).

\section{Acknowledgements}

The authors are grateful to the reviewers for their critical comments and valuable suggestions.

\section{References}

1. F. Harary, Graph Theory (Addison Wesley, Reading, Mass, 1969).

2. B. Basavanagoud, J. Discrete Math. Sci. Cryptography 1, 133 (1998).

3. D.V.S.Sastry and B.Syam Prasad Raju, Discrete Mathematics 48, 113 (1984). doi:10.1016/0012-365X(84)90137-7

4. V.R.Kulli and B.Basvanagoud, Bull. Calcutta Math. Soc. 94, 1 (2002).

5. H. P. Patil and V. R. Kulli, J. Discussiones Mathematicae 7, 97 (1985). 